\title{
Connexin30-mediated intercellular communication plays an essential role in epithelial repair in the cochlea
}

\author{
Andrew Forge*, Daniel J. Jagger, John J. Kelly ${ }^{\ddagger}$ and Ruth R. Taylor \\ Centre for Auditory Research, UCL Ear Institute, London WC1X 8EE, UK \\ ${ }^{\ddagger}$ Present address: Department of Anatomy and Cell Biology, Schulich Dental Science Building, University of Western Ontario, London, ON N6A 5C1, Canada \\ *Author for correspondence (a.forge@ucl.ac.uk) \\ Accepted 28 January 2013 \\ Journal of Cell Science 126, 1703-1712 \\ (c) 2013. Published by The Company of Biologists Ltd \\ doi: 10.1242/jcs. 125476
}

\section{Summary}

A role for connexin $(\mathrm{Cx}) 30$ in epithelial repair following injury was examined in the organ of Corti, the sensory epithelium of the cochlea. In this tissue, lesions caused by loss of the sensory hair cells are closed by the supporting cells that surround each one. Gap junctions in which $\mathrm{Cx} 30$ is the predominant connexin are large and numerous between supporting cells. In mice carrying a deletion in the gene (Gjb6) that encodes Cx30, the size and number of gap junction plaques, and the extent of dye transfer, between supporting cells was greatly reduced compared with normal animals. This corresponded with unique peculiarities of the lesion closure events during the progressive hair cell loss that occurs in these animals in comparison with other models of hair cell loss, whether acquired or as a result of a mutation. Only one, rather than all, of the supporting cells that contacted an individual dying hair closed the lesion, indicating disturbance of the co-ordination of cellular responses. The cell shape changes that the supporting cells normally undergo during repair of the organ of Corti did not occur. Also, there was disruption of the migratory activities that normally lead to the replacement of a columnar epithelium with a squamous-like one. These observations demonstrate a role for Cx30 and intercellular communication in regulating repair responses in an epithelial tissue.

Key words: Cochlea, Connexin, Cx30, Gap junction, Wound healing

\section{Introduction}

Gap junction channels are composed of hexamers of the protein, connexin. There are 21 connexin genes in the human genome and 20 in that of the mouse (Bedner et al., 2012). There is some understanding of the varying biophysical properties of channels of different connexin composition (Kanaporis et al., 2011; Manthey et al., 2001; Yum et al., 2007) but the functional significance of expression of a particular connexin in a tissue is less clear.

In the organ of Corti, the sensory epithelium of the cochlea, each sensory 'hair' cell is surrounded and separated from its neighbours by the non-sensory supporting cells, and supporting cells are extensively coupled by numerous, unusually large gap junctions (Forge et al., 2003; Jagger and Forge, 2006). The gap junctional channel proteins that are localized to these cells are connexin $26(\mathrm{Cx} 26)$ and connexin 30 (Cx30) and several cell types co-express Cx26 and Cx30. In vitro studies using cell lines have shown that $\mathrm{Cx} 26$ and Cx30 can oligomerize to form heteromeric channels (Marziano et al., 2003; Yum et al., 2007) and there is evidence that $\mathrm{Cx} 26 / \mathrm{Cx} 30$ heteromeric channels exist in the cochlea (Ahmad et al., 2003; Forge et al., 2003; Jagger and Forge, 2006). However, there are regions where Cx26 is the predominant connexin and others where $\mathrm{Cx} 30$ predominates (Jagger and Forge, 2006). In the functionally mature organ of Corti of mice, Cx30 is the predominant connexin in the gap junction plaques between apposed Deiters' cells, the supporting cells that surround the outer hair cells (OHC) (Jagger and Forge, 2006; Sun et al., 2005).
During sound-induced mechanotransduction, hair cells modulate a $\mathrm{K}^{+}$current that flows through them from the $\mathrm{K}^{+}$-rich (endolymphatic) fluid that bathes the luminal surface of the sensory epithelium to the $\mathrm{K}^{+}$-poor extracellular spaces that surround the bodies of the hair and supporting cells. The supporting cells are thought to buffer the extracellular $\mathrm{K}^{+}$to maintain a low $\mathrm{K}^{+}$concentration around the hair cell body. $\mathrm{K}^{+}$is redistributed away from the sensory region via an intracellular route involving gap junction mediated intercellular communication (Wangemann, 2002). However, channels composed of the connexins present in the organ of Corti do not especially favour $\mathrm{K}^{+}$transfer in comparison with channels composed of other members of the connexin family. Thus, it seems likely that the particular connexins expressed in the organ of Corti support specific activities in addition to $\mathrm{K}^{+}$buffering, and supporting cells have other roles.

When OHC die, the Deiters' cells can act as phagocytes to take up the cellular debris (Abrashkin et al., 2006; Forge, 1985; Taylor et al., 2008). Also, when hair cells are lethally injured the supporting cells expand to close the lesion created by the dying cell in a manner that preserves tight junctional permeability barriers at the luminal surface of the sensory epithelium (Forge, 1985; McDowell et al., 1989; Raphael and Altschuler, 1991). This lesion closure involves a coordinated response by all those supporting cells that surround an individual hair cell and results in a stereotypical, regular pattern of cells at the apical surface of the epithelium (Forge, 1985; Hawkins and Engstrom, 1963; Raphael and Altschuler, 1991). How the co-ordination of cell responses is achieved is not known but the presence of numerous large gap junction plaques between Deiters' cells suggests the possibility that some of the necessary intercellular signalling 
might be conveyed by gap junctions. In skin, gap junctions are thought to be important for co-ordinating cellular responses during wound healing (Kretz et al., 2003) and it has been proposed that $\mathrm{Cx} 30$ may play a crucial role (Coutinho et al., 2003) in those events.

Mice with ablation of the gene (Gjb6) that encodes $\mathrm{Cx} 30$ (Cx30 null mice) are long-lived, breed and show no obvious abnormalities except they are profoundly deaf from the usual time of hearing onset [postnatal day (P)12] and undergo progressive OHC loss beginning at around P15-P16 (Sun et al., 2009; Teubner et al., 2003). The availability of these mice affords an opportunity to test the hypothesis that intercellular communication mediated by $\mathrm{Cx} 30$ plays an important role in lesion repair responses in an epithelial tissue. Here we use the Cx30 null mice to examine the effects of absence of Cx30 upon gap junctions and intercellular communication in the mature organ of Corti and upon repair of the sensory epithelium following hair cell loss.

\section{Results}

In normal mice, the onset of hearing is around $\mathrm{P} 12$ by which time the organ of Corti is structurally mature. Although Cx30 null mice are deaf at P12 (Teubner et al., 2003), their organs of Corti appeared to reach structural maturity at around the same age (Fig. 1A). This indicates that loss of $\mathrm{Cx} 30$, which in normal mice is first detectable in the organ of Corti at about P8-P10 (Jagger and Forge, 2006; Qu et al., 2012), does not obviously affect organ of Corti development.

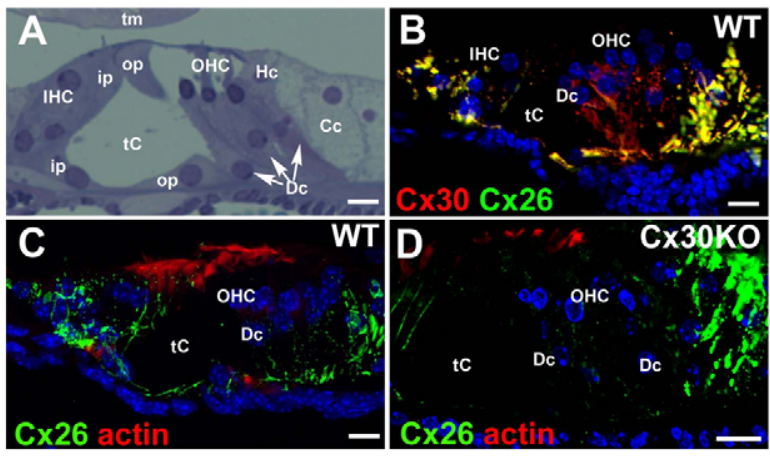

Fig. 1. Connexin labelling in the organ of Corti. (A) Toluidine Blue stained section of the organ of Corti of Cx30 null mouse at P14. The organ of Corti displays the normal, mature structure. (B) Immunolabelling for Cx30 (red) and Cx26 (green) in organ of Corti of wild-type (WT) animal. Labelling for Cx30 predominates in the Deiters' cell region. Intense labelling for Cx26 and Cx30 amongst Hensen's and Claudius' cells to the lateral side and amongst the cells around and medial to inner hair cells. (C) Cx26 labelling (green) in the wild-type organ of Corti. Phalloidin labels actin (red) at the apical ends of hair cells and supporting cells. Some small plaques labelled for $\mathrm{Cx} 26$ are present in the Deiters' cell region. (D) Cx26 labelling in Cx30 null organ of Corti. Some small plaques of Cx26 labelling are still evident in the Deiters' cell region and Cx26 is highly expressed in the Hensen's cells and other cells on the lateral side as well as in the cells to the medial side of the tunnel of Corti. IHC, inner hair cell; OHC, outer hair cell; Dc, Deiters' cell; Hc,

Hensen's cell; Cc, Claudius' cell; op, outer pillar cell; ip, inner pillar cell; tm, tectorial membrane; $\mathrm{tC}$, tunnel of Corti. Scale bars: $10 \mu \mathrm{m}$.

\section{Connexin expression pattern in the organ of Corti}

In WT animals, immunolabelling both of whole mount preparations (not shown) and frozen sections (Fig. 1B) of the organ of Corti showed Cx30 to be the predominant connexin expressed amongst Deiters' cells. Labelling for Cx26 was weak in this location, but there was intense labelling for $\mathrm{Cx} 26$ in cells either side of the Deiters' cell/OHC region (Fig. 1B,C) usually together with $\mathrm{Cx} 30$ (Fig. 1B). Cx26 labelling often appeared to be predominant on inner pillar cells and Hensen's cells. In the organ of Corti of $\mathrm{Cx} 30$ null animals there was also very little labelling for Cx26 amongst Deiters' cells, although some small plaques of Cx26 positive labelling were evident (Fig. 1D), indicating that the loss of $\mathrm{Cx} 30$ is not compensated for by any significant upregulation of $\mathrm{Cx} 26$. Elsewhere the labelling for Cx26 appeared similar to that in the organ of Corti of WT animals (Fig. 1C). To determine whether loss of $\mathrm{Cx} 30$ might be compensated for by expression of another connexin, the cochleae of Cx30 null animals were immunolabelled for $\mathrm{Cx} 43 . \mathrm{Cx} 43$ is expressed in the organ of Corti in the earliest stages of its differentiation, but is downregulated at early post-natal ages and disappears from the organ of Corti prior to the age at which expression of $\mathrm{Cx} 30$ is initiated (Cohen-Salmon et al., 2004). Labelling for $\mathrm{Cx} 43$ in the mature cochlea of $\mathrm{Cx} 30$ null animals was the same as that in control animals (Forge et al., 2003): it was confined to the extreme lateral edge of the spiral ligament but was absent from the organ of Corti (not shown).

\section{Gap junctions in Cx30 null mice}

In thin sections of the organ of Corti of control animals, the plasma membranes of the cell bodies of adjacent Deiters' cells, below the level of the $\mathrm{OHC}$, were parallel and closely apposed, and there were large gap junctions running a considerable length along the appositions (Fig. 2A). In the Cx30 null animals there were significant separations of the apposed membranes of Deiters' cell bodies interspersed with a few short 'kiss-like' segments of close apposition (Fig. 2B) some of which exhibited gap-junction-like structure (Fig. 2B, inset). Sometimes the separations of the cell bodies were quite wide affecting the majority of the region of apposition between adjacent cell bodies (Fig. 2B). These structural features were evident in regions where all hair cells were present at all ages examined from P14 to 3 months, between cells of all three rows of Deiters' cells and at all locations along the cochlear spiral. In conditionally Cx26 deficient mice, in which $\mathrm{Cx} 26$ is absent from the organ of Corti (Wang et al., 2009), maturation of supporting cells is delayed, but a near mature structural condition is apparent where hair cells persist at 60 days after birth, particularly in the apical half of the cochlea. In thin sections of the organ of Corti of conditionally Cx26 deficient animals of this age (Fig. 2D) appositions between the membranes of adjacent Deiters' cell bodies appeared similar to those in control animals. Plasma membranes were closely apposed and parallel with no separations, and there were long gap-junction-like structures extending the length of the appositions. These observations confirm that deficiency in $\mathrm{Cx} 30$ results in a specific change in the intercellular relationships between Deiters' cells.

Freeze-fracture revealed effects on the size and number of gap junctions between Deiters' cells with absence of Cx30. In freezefracture replicas gap junctions appear as plaques of closely packed particles, ca.10-12 nm diameter on the fracture face of that plasma membrane leaflet that apposes the cytoplasm (the 'p'-face), or as 

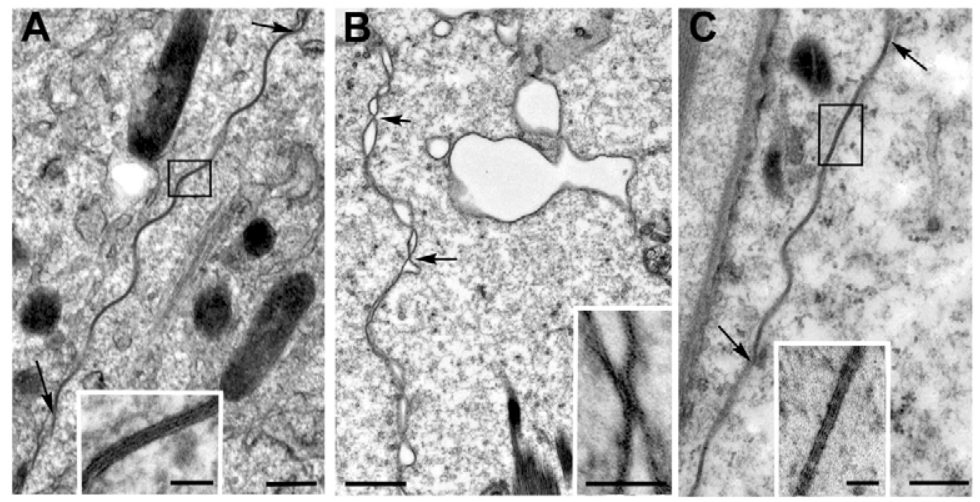

Fig. 2. Deiters' cells in thin sections. (A) P25 control mouse. Plasma membranes of the adjacent cells are closely parallel with no space between. Inset shows higher magnification of a region of the apposition (boxed) that reveals the typical septilaminar appearance of a gap junction plaque in section. Arrows indicate limits of gap-junction-like structure. (B) Cx30 null mice at P17. There are numerous separations of the adjacent plasma membranes. Where the membranes meet over short distances (arrows), there is no space between them as in gap junction plaques. Inset shows region of close apposition at higher magnification in tissue from P30 animal; the appearance is similar to that of a gap junction. (C) Cx26 conditional knockout mouse at P60. Adjacent membranes are closely parallel with no space between them. Arrows indicate limits of gap-junction-like structure. Inset shows detail of gapjunction-like structure in the boxed region of the apposition. Scale bars: $0.5 \mu \mathrm{m}$ (A), $50 \mathrm{~nm}$ (inset A); $1 \mu \mathrm{m}$ (B), $0.25 \mu \mathrm{m}$ (C).

small pits on the fracture face of the plasma membrane leaflet that apposes the extracellular space ('e'-face). These particles (or pits) represent the gap junctional channel units (connexons). In the organs of Corti of control mice, gap junction plaques on the fracture faces of the membranes of Deiters' cells were numerous and large, of a size and number consistent with those previously described for normal animals of several different species (Forge et al., 2003) (Fig. 3A,B). In Cx30 null animals some gap junction plaques were still evident upon the fracture faces of Deiters' cell bodies, but these were small (Fig. 3C-E), often consisting of no more than two or three short rows of connexons (Fig. 3E). The plaques were dispersed along the membranes at separations approximately similar to those of the kiss-like appositions revealed in thin sections (Fig. 2B,C). In contrast to the changes in gap junction plaques evident amongst Deiters' cells, gap junctions associated with Hensen's cells, those cells immediately adjacent to the Deiters' cells on the outer side of the organ of Corti, were similar in size to those associated with this cell type in normal animals (Forge et al., 2003) (Fig. 3F). Gap junctions in the Hensen's cell region are likely to be formed of homomeric Cx26 channels and heteromeric Cx26/Cx30 channels (Fig. 1B) (Jagger and Forge, 2006; Sun et al., 2009). The fracture faces of the plasma membranes of Hensens' cells were identified by the presence on them of square arrays of particles (or pits) characteristic of aquaporin 4 that has been localized to that cell type (Hirt et al., 2011).
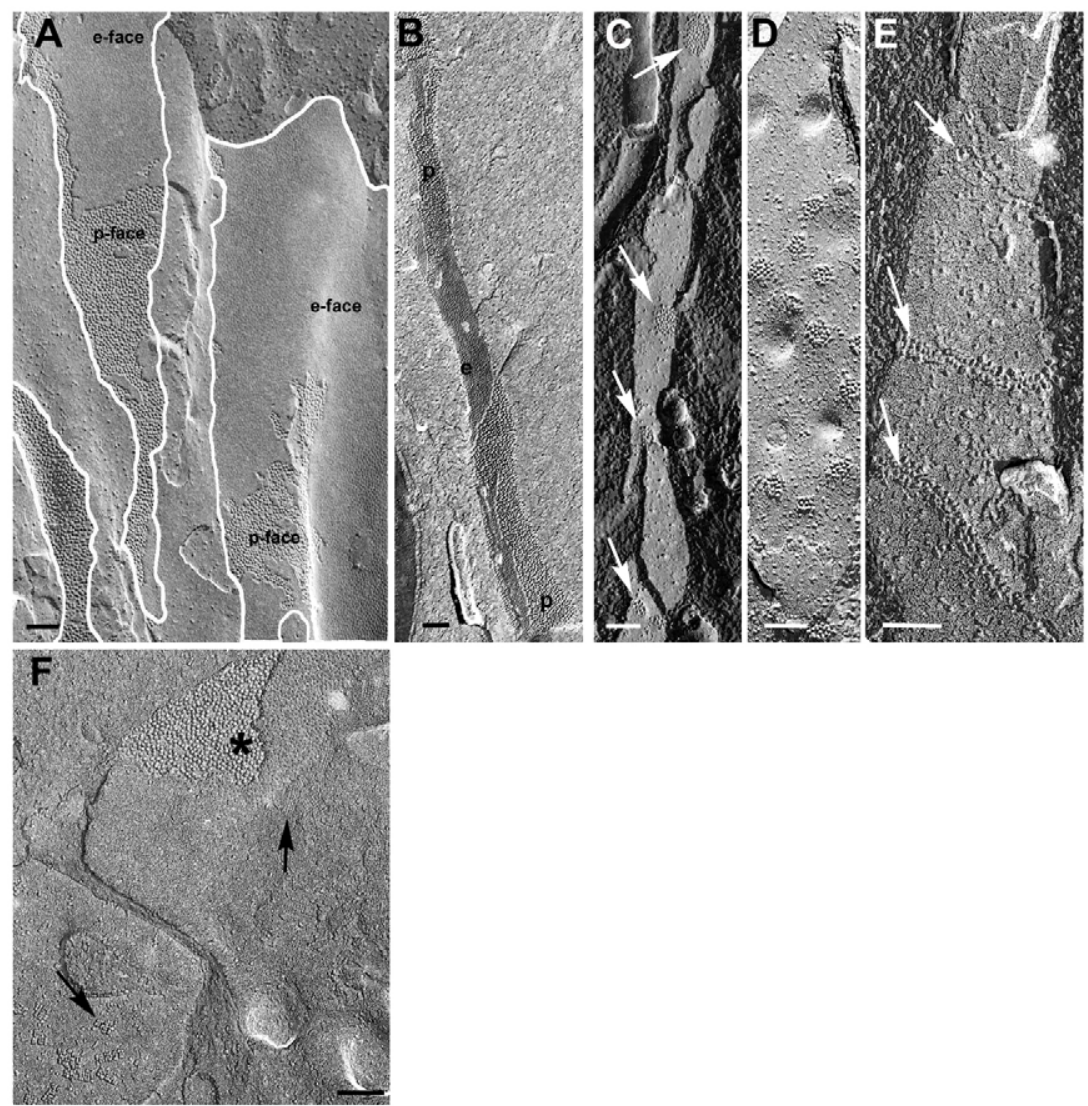

Fig. 3. Gap junction plaques exposed by freeze-fracture. (A,B) Plasma membranes of wild-type Deiters' cells. (A) Full face view: the gap junction plaques are outlined. The plaques are very large and appear as closely packed particles on the protoplasmic face or pits on the exoplasmic face where the fracture plane has jumped from the membrane of one cell to that of the other with which it is coupled. (B) Partially side-on view of the membrane. The gap junction plaque extends the full length of the membrane profile. Exoplasmic and protoplasmic faces are indicated. (C-E) Deiters' cells in Cx30 null mouse. (C,D) Similar views of the membrane fracture faces as shown in $\mathrm{A}$ and $\mathrm{B}$, respectively. There are several small plaques (indicated by arrows in C), each consisting of a small number of connexons. (E) Arrows point to rows of closely packed particles of a size equivalent to connexons, indicating small linear junction plaques. (F) Hensen's cell in Cx30 null mouse. A large gap junction plaque is indicated by the asterisk. The arrows indicate square arrays of particles and pits that are a characteristic of the membrane fracture faces of Hensen's cells. e, exoplasmic; p, protoplasmic. Scale bars: $100 \mathrm{~nm}(\mathrm{~A}-\mathrm{E}), 200 \mathrm{~nm}$ (F). 


\section{Intercellular communication between supporting cells in the organ of Corti}

We have shown previously (Jagger and Forge, 2006; Taylor et al., 2012) that in the normal organ of Corti there is extensive gap junctional communication between the supporting cells, and that in the functionally mature organ of Corti, there are two coupled compartments. One consists of all the supporting cells from the inner pillar cells inwards; the other consists of outer pillar cells, Deiters' cells and all those cells to the outer side. Thus, when neurobiotin (Nbn) was injected into an individual Deiters' cell (Fig. 4B) it spread radially to all other Deiters' cells, to Hensen's, cells and all the cells on the outer side of the organ of Corti as well as inwards to the outer pillar cells. However, it did not cross to the inner pillar cell. The dye also spread from the injected Deiters' cell to many other cells longitudinally. Likewise dye injected into a single Hensen's cell (not shown) transferred radially to Deiters' cells and to the cells to the outer side, as well as longitudinally.

In the structurally mature organ of Corti of Cx30 null animals at P15-P16, dye transfer amongst Deiters' cells was severely restricted. Nbn injected into a single Deiters' cell spread to only a very few other Deiters' cells (Fig. 4C), consistent with the reduced size and number of gap junction plaques on Deiters' cells observed by freeze-fracture. Furthermore the tracer did not
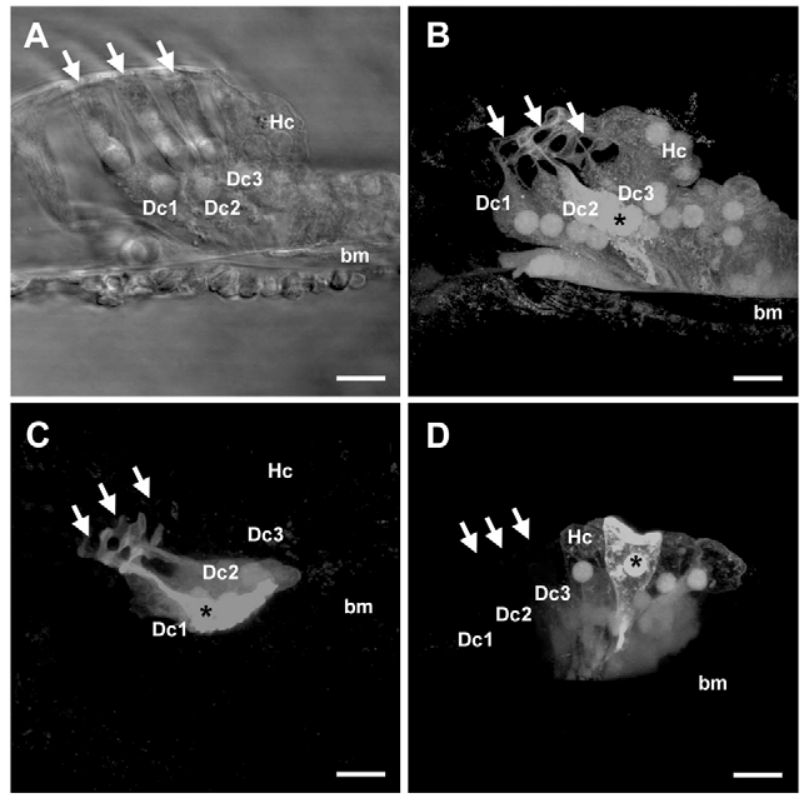

Fig. 4. Gap junctional dye transfer is restricted in cochlear slice preparations from Cx30 null mice. (A) DIC image of organ of Corti at P15. Arrows indicate OHC. (B) Distribution of neurobiotin (Nbn) after its injection into a single P15 Deiters' cell is indicated by the asterisk. Nbn transfers to all other Deiters' cells and in to the outer pillar cell, as well as to Hensen's cells and the cells to the outer side. Dye does not pass to OHC, the positions of which are indicated by the arrows, demonstrating that hair cells are not coupled to supporting cells. (C,D) Cochlear slice from Cx30 null mouse at P15. (C). Nbn injected into a single Deiters' cell (indicated by the asterisk) transfers to only a few other Deiters' cells. It does not transfer to Hensen's cells. (D) Nbn injected into a single Hensen's cell (indicated by the asterisk) transfers to many other Hensen's cells and to the other cells on the lateral side, but it does not transfer medially to Deiters' cells. Dc1-Dc3, Deiters' cells; Hc, Hensen's cell; bm, basilar membrane. Scale bars: $10 \mu \mathrm{m}$. transfer from the Deiters' cell to Hensen's cells (Fig. 4C). Similarly, dye injected into a single Hensen's cell did not transfer to Deiters' cells (Fig. 4D). It did however, spread extensively to other Hensen's cells and the other cells to the outer side of the organ of Corti, in line with the continued presence of large gap junction plaques between these cell types as observed by freezefracture and immunolabelling for $\mathrm{Cx} 26$. These dye transfer studies indicate that loss of $\mathrm{Cx} 30$ from the mature organ of Corti significantly reduces intercellular communication between Deiters' cells and prevents coupling of the Deiters' cell population with Hensen's cells.

\section{Normal pattern of cellular repair at the apical surface of the organ of Corti}

At the apical surface of the undamaged organ of Corti of normal mice, there is a regular mosaic of cells, the so-called reticular lamina, in which each hair cell is separated from its neighbour by intervening supporting cells (Fig. 5A). When hair cells are lost the spaces they once occupied are filled by expansion of the heads of the supporting cells that surround each one. This produces a stereotypical pattern of cellular repair at the reticular lamina that is seen in almost all situations where $\mathrm{OHC}$ are lost. This pattern was displayed in the organs of Corti of control littermates of $\mathrm{Cx} 30$ null mice that had been treated with a combination of the loop diuretic, bumetanide, and kanamycin, an aminoglycoside antibiotic (Fig. 5B), a treatment regime which causes loss of almost all OHC within 48 hours (Taylor et al., 2008; Taylor et al., 2012). At the reticular lamina, each OHC in the first (innermost) row was replaced by the expanded heads of three cells ( 2 adjacent outer pillar and single 1st row Deiters' cells); each $\mathrm{OHC}$ in the second row was replaced by 4 supporting cells (outer pillar and three adjacent Deiters' cells); and each third row $\mathrm{OHC}$, was also replaced by four cells (4 adjacent Deiters' cells). The head of the inner pillar cell although normally in contact with the first row $\mathrm{OHC}$ did not participate in the repair. Rather the inner pillar cell head retracted to expose the head of the underlying outer pillar cell (arrow in Fig. 5B), a feature commonly seen in most conditions where $\mathrm{OHC}$ are lost (Taylor et al., 2012), regardless of cause, for example with mutations that are associated with hair cell loss such as those in Ptprq (Goodyear et al., 2003) (Fig. 5C).

\section{Aberrant lesion closure in $\mathbf{C} \times 30$ null animals}

The Cx30 null mice lose OHC progressively (Teubner et al., 2003; Sun et al., 2009). At ca. 15-17 days after birth at the basalmost end of the organ of Corti there was scattered loss of $\mathrm{OHC}$ but most $\mathrm{OHC}$ were still present in the upper part of the basal coil. OHC loss had spread to the upper middle coil by 2 months of age. The heads of supporting cells filled the spaces once occupied by hair cells but the pattern of cellular repair in the Cx30 null mice was different from that which occurs following hair cell loss in normal animals.

In the organs of Corti of $\mathrm{Cx} 30$ null mice, the lesions created at the apical surface by the 'natural' (uninduced) hair cell loss were repaired by supporting cells, but the cellular pattern was irregular. The sites of loss of individual $\mathrm{OHC}$ were often closed by only one of the adjacent supporting cells rather than several of them (Fig. 5D-I). Also, inner pillar cells participated in closure. Thus, the space from which an $\mathrm{OHC}$ in the first row was missing was often replaced at the surface by an extension of the head of an inner pillar cell, that extension filling the entire 

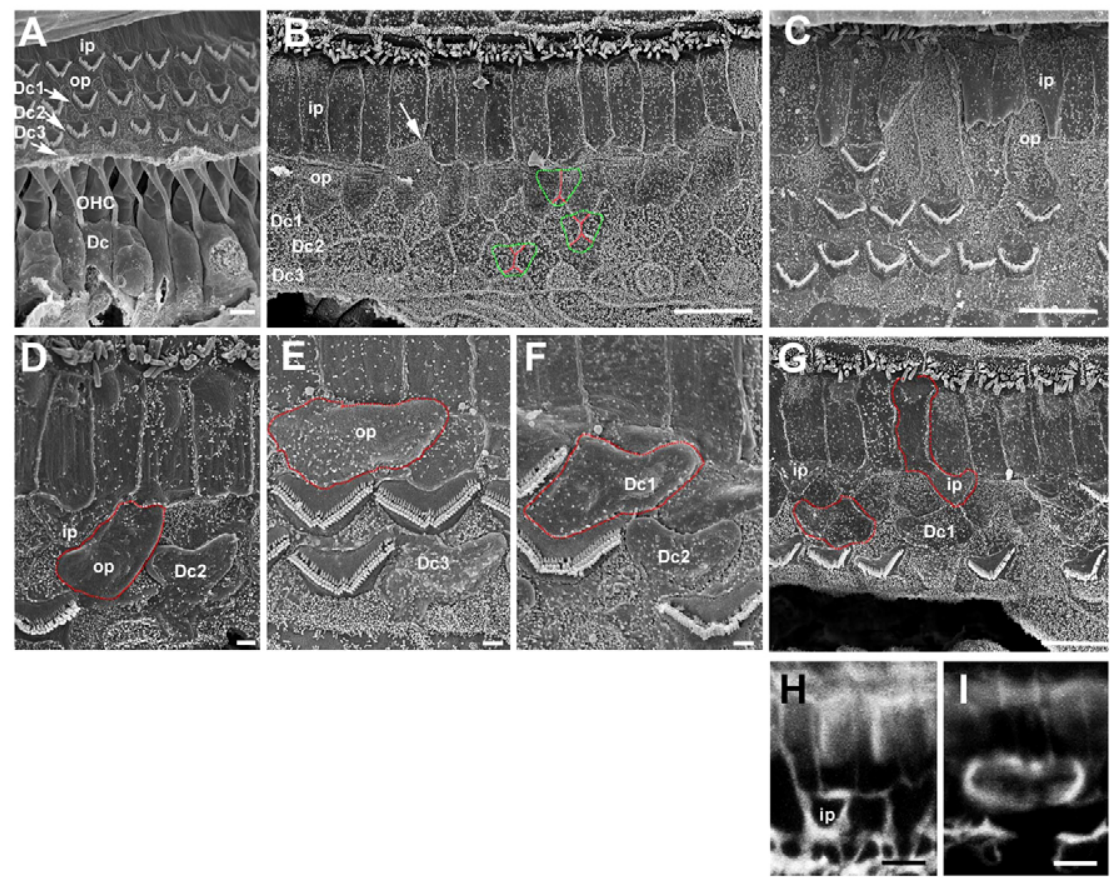

Fig. 5. Aberrant repair by supporting cells following hair cell loss in Cx30 null mice. (A) Normal, undamaged organ of Corti in control mouse. There are three rows of $\mathrm{OHC}$, each one separated from its neighbour by the intervening heads of supporting cells. The cells to the outer side have broken away to reveal the body of the organ of Corti. Dieters' cells consist of a cell body region, and a thin phalangeal process that rises up to the luminal surface where the head expands to fill the space between OHC. Within the body of the organ of Corti are large extracellular spaces around the body of the OHC and the phalangeal processes of the supporting cells. (B,C) Stereotypical pattern of repair in organ of Corti following hair cell loss in control mice. (B) Repaired organ of Corti following OHC loss induced by combined administration of kanamycin and bumetanide (48 hours post-treatment). Stereotypical regular mosaic-like pattern of cells at the luminal surface is created by expansion of the heads of supporting cells into the sites from which OHC have been lost. The position where an OHC in each row once was is outlined in green. The participation of the supporting cells that surround each $\mathrm{OHC}$ in the lesion closure is outlined in red. Arrow indicates initiation of retraction of the heads of inner pillar cells exposing the apical surface of the outer pillar cell. (C) Retraction of heads of inner pillar cells in Ptprq mutant mouse. (D-G) Repair in Cx30 null mice. The site of a lost OHC is filled by the head of only a single supporting cell. The border of the head of the supporting cell effecting lesion closure is outlined in some instances. Examples of the head of the inner pillar cell replacing 1st row OHC are shown in D and G. There is no consistency in which of the supporting cells that contacted an OHC in a particular row expands to fill the space. (H,I) Phalloidin labeling of F-actin outlining the cell junctions. (H) Continuity of the cell border of an inner pillar cell that has expanded into the space of a lost first row OHC. (I) Single 1st row Deiters' cell that expanded to replace an OHC in the second row. Dc1-Dc3, Deiters' cells in rows 1, 2 and 3; ip, inner pillar cell; op, outer pillar cell. Scale bars: $10 \mu \mathrm{m}$

(A-C), $2 \mu \mathrm{m}(\mathrm{D}-\mathrm{F}), 10 \mu \mathrm{m}(\mathrm{G}-\mathrm{I})$.

space, and taking up the shape, of the OHC that had been lost (Fig. 5D,G,H). In other instances a first row $\mathrm{OHC}$ was replaced by the head of a single outer, rather than inner, pillar cell (Fig. 5E). Second row OHC could be replaced by the head of a single outer pillar cell or the head of a single first row Deiters' cell; individual second row $\mathrm{OHC}$ could be replaced by a single first row Deiters' cell or a single second row Deiters' cell; and third row $\mathrm{OHC}$ by individual second or third row Deiters' cell (Fig. 5D-G). The use of phalloidin to label F-actin associated with the adherens junctional complexes at the luminal end of the cells outlined the head of each cell. This confirmed that only a single supporting cell head expanded into the space of the lost OHC (Fig. 5H,I). It also showed continuity of the junctions, suggesting maintenance of junctional complexes during the cellular re-organisation at the apical surface. In line with this inference, no obvious lesions through the apical surface were evident by SEM or in thin sections.

Changes in Deiters' cell body shape following hair cell loss In control mice treated with kanamycin-bumetanide, and typical following loss of $\mathrm{OHC}$ in most conditions, in addition to expansion of the heads of the supporting cells at the surface, within the body of the organ of Corti, the normally thin phalangeal processes of the Deiters' cells enlarged to fill the prominent extracellular spaces that surround them such that adjacent Deiters' cells came closely adjacent along much of their length rather than just around the cell body region (e.g. Taylor et al., 2012) (Fig. 6A,B). Subsequently the columnar supporting cells, Deiters' and pillar cells, may become replaced by a squamous-like 'flat' epithelium formed by cells from the outer side of the sensory strip migrating across the basilar membrane (Taylor et al., 2012). This epithelial re-organisation is typical of many conditions in which hair cells are lost and was observed in the organs of Corti both of transgenic mice carrying the R75W mutation in CX26 and mice with $\mathrm{Cx} 26$ conditionally ablated from the organ of Corti (Fig. 6C).

In the $\mathrm{Cx} 30$ null mice, in addition to peculiarities of lesion closure at the apical surface of the organ of Corti, the phalangeal processes of the Deiters' cells did not widen during the tissue repair that followed hair cell loss (Fig. 6D,E). Deiters' cells retained a morphology similar to that in undamaged tissue (Fig. 5A) and at no time up to 16 months of age, the oldest 

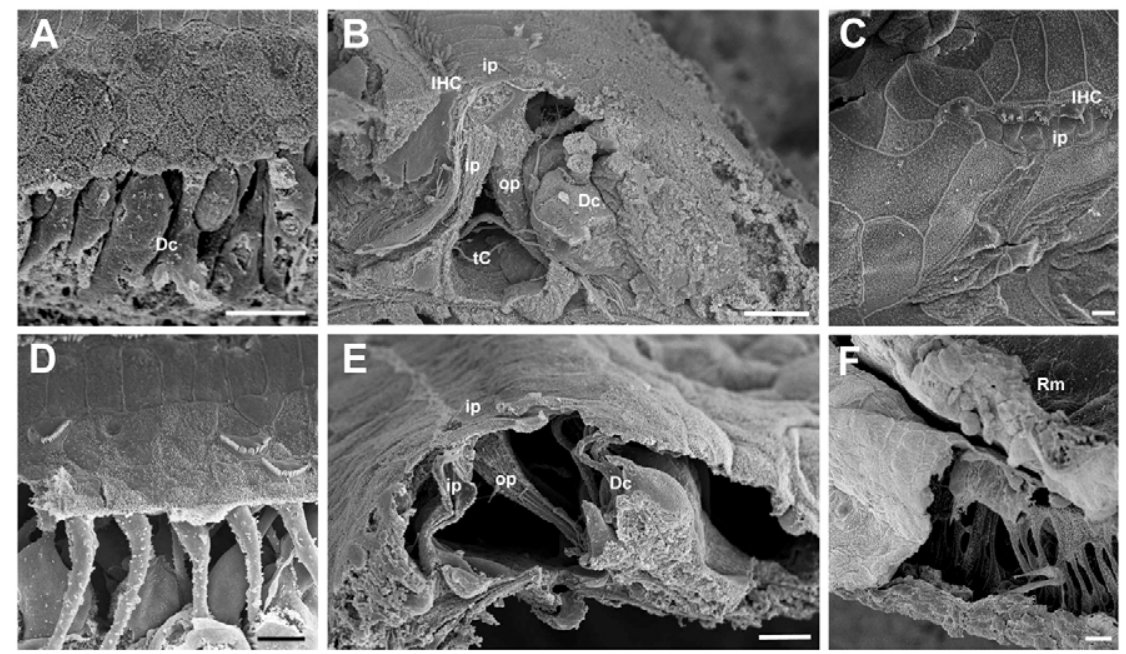

Fig. 6. Lack of supporting cell expansion following hair cell loss in Cx30 null mice. (A,B) Control mouse. The body of the organ of Corti after hair cell loss (48 hours following kanamycin-bumetanide treatment). (A) Viewed from the lateral side, cells on the outer side have broken away revealing bodies of Deiters' cells. (B) Radial view of a break across the organ of Corti. The phalangeal processes of Deiters' cells have widened and the large extracellular spaces normally present in the undamaged tissue have been occluded. (C) Conditional Cx26 knockout mouse. Surface view of the flat, squamous-like epithelium generated by migration of cells from the outer-side of the organ of Corti across the region normally occupied by the columnar supporting cells. Some heads of surviving inner pillar cells, and some surviving inner hair cells are evident. (D-E) Cx30 null mice. Views of the body of the organ of Corti, similar to those shown in A and B, after hair cell loss, at 2 months old (D) and 6 months old (E). There is no expansion of the phalangeal processes of Deiters' cells and the large extracellular spaces remain. (F) Cx30 null mice. Views of the body of the organ of Corti, similar to those shown in E, at 6 months of age. The layer of cells to the outer side of organ of Corti has moved upwards to approach the Reissner's membrane, creating enlarged extracellular spaces within the organ of Corti, in which the thin phalangeal processes of supporting cells persist. IHC, inner hair cell; ip, inner pillar cell; op, outer pillar cell; Rm, Reissner's membrane; tC, tunnel of Corti. Scale bars: $10 \mu \mathrm{m}$.

examined, was there closure of the extracellular spaces within the organ of Corti in regions of total hair cell loss. Nor was there formation of a squamous-like, flat epithelium. Rather, from about 6 months of age, where all $\mathrm{OHC}$ were lost, there was initially gross enlargement of the extracellular spaces within the organ of Corti, most prominently that between the third row Deiters' cell and the cells to the outside (Fig. 6F) that was most obvious in sections (Fig. 7A,B). The fragility of the tissue in this condition made preservation for SEM difficult. The enlargement resulted from a heightening of the organ of Corti in the region outside the third row of Deiters' cells. The luminal surface of the enlarged extracellular space was covered by flattened cells in a single layer continuous with cells of the outer sulcus. These flattened cells showed morphological characteristics similar to those of the relatively non-specialized cells that normally reside to the outside of the Deiters' cells but they were detached from the basilar membrane (Fig. 7A,B).

The heightening of the outer region of the organ of Corti was accompanied by a re-orientation of the apical surface of the epithelium across the Deiters' cell region relative to the underlying basilar membrane from the usual parallel orientation to an almost perpendicular one. Deiters' cells, however, remained in place and attached to the basilar membrane; their cell bodies showed normal morphology; and their apically projecting phalangeal processes were thin but reached to the epithelial surface (Fig. 7B): the elaborate junctional complexes characteristically associated with the neck region between adjacent cells at the luminal end of supporting cells were still evident in the distorted organ of Corti identifying the head regions of the Deiters' cells and indicating preservation of intercellular junctions (Fig. 7C). The pillar cells also remained in place and attached to the underlying basilar membrane appearing morphologically normal (Fig. 7B). Their characteristic prominent microtubule bundles maintained a normal arch and tunnel of Corti such that the 'upward' (luminal side) bend in the epithelium that resulted in the perpendicular orientation of the reticular lamina was created by a folding at the level of the outer pillar cells. At this stage the surface of the epithelium from the inner pillar cell inwards remained parallel to the basilar membrane and the cells of the inner sulcus remained attached to the basilar membrane and bony lip. This morphology of the organ of Corti in Cx30 null mice with OHC loss gave the impression of inward movement of the layer of cells to the outerside of the organ of Corti without accommodating movements of Deiters' or pillar cells which by remaining rigidly in place caused upward deflection of the inward migrating cell layer thereby generating the observed folding.

Where the organ of Corti was distorted, the tectorial membrane (the extracellular matrix material that overlies the organ of Corti) was displaced (Fig. 7A) and became detached from its normal anchorage at the spiral limbus (Fig. 7D). It often became rounded up, and its fibrillar structure was disorganized (Fig. 7E). Unusually, the detached tectorial membrane became surrounded by a single layer of cells, which appeared distinct and separated from the nearby Reissner's membrane (Fig. 7E). Immunollabelling showed the cells surrounding the tectorial membrane to express $\mathrm{C} \times 26$ in gapjunction-like plaques (Fig. 7F). This suggests these cells derived from the non-specialized cells of the organ of Corti and not from the Reissner's membrane, the cells of which normally do not express any connexin.

In the cochleae of older Cx30 null animals, 14-16 month of age, the layer of cells arising from and continuous with the outer 


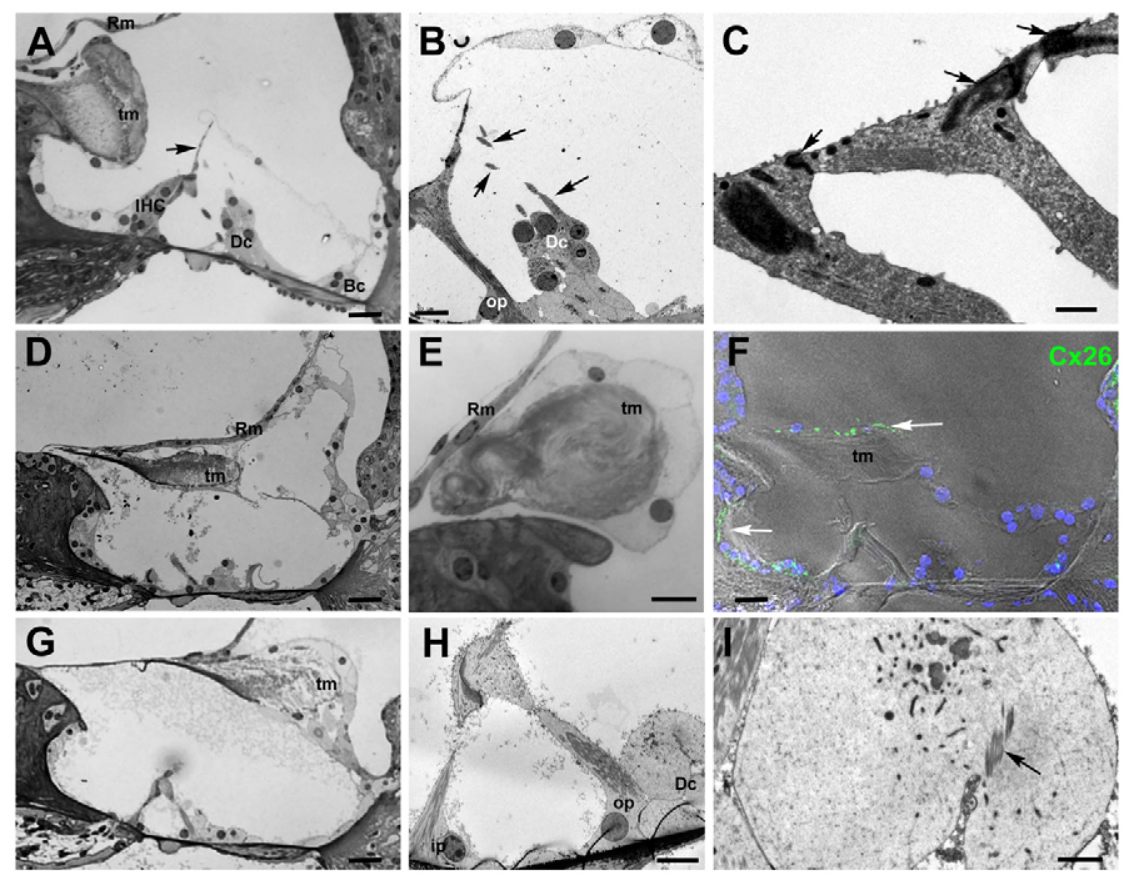

Fig. 7. Disruption of the organ of Corti in older Cx30 null mice. (A) Toluidine-Blue-stained section from a 6-month-old mouse. Large extracellular space to the lateral side of the organ of Corti is covered by supporting cells detached from the basilar membrane, but Boettcher's cells and other cells to the outer side remain in place. The apical surface across the Deiters' and pillar cells re-oriented perpendicular to the basilar membrane (arrow). The tectorial membrane is rounded up and displaced. A surviving IHC is evident. Reissner's membrane appears largely normal. (B) Thin section close to the area in A. Outer pillar cell has normal morphology with bundle of closely packed microtubules. Deiters' cells also appear normal, with thin phalangeal processes (arrows indicate sections through phalangeal processes) with no shape change. (C) The apical end of the Deiters' cells in region close to that in A and B. Arrows indicate the (intact) junctions between cells where there are accumulations of microfilaments running quite deeply down the depth of the junction, a specialization of the junctions associated with the supporting cells of the organ of Corti. (D) Section from 15-month-old mouse. The tectorial membrane is detached from its anchoring on the spiral lamina. The cells to the outer side of the organ of Corti, detached from the basilar membrane, form a continuous sheet that stretches up to Reissner's membrane, but recognizable pillar cells and Deiters' cells remain in place. (E) The tectorial membrane is rounded up and surrounded by a cell monolayer that is separate from Reissner's membrane. The fibrils that form the tectorial membrane are disorganized. (F) Merged DIC and fluorescence images showing presence and location of immunofluorescence for Cx26 (arrows). Labelling is present at borders between the cells that surround the tectorial membrane and in cells of the inner sulcus. (G) Section from 16-month-old mouse. The cells on the outer side of the organ of Corti, detached from the basilar membrane, reach to Reissner's membrane and surround the tectorial membrane, which has moved across to the lateral side of the scala media and is highly disorganized. Pillar and Deiters' cells remain in place. (H) Inner and outer pillar cells in the region shown in G. Both have normal morphology with organized bundles of closely packed microtubules that maintain the tunnel of Corti. The luminal end of the cells is sealed by a plasma membrane. Deiters' cells are rounded, but retained on the basilar membrane. (I) Deiters' cell in region of G and H. Other than the rounding, the morphology is normal with no evidence of any cellular degeneration. The arrow indicates microtubule bundle, an identifying characteristic of Deiters' cells. Bc, Boettcher's cells; Dc, Deiter's cell; Rm, Reissner's membrane; tm, tectorial membrane; ip, inner pillar cell; op, outer pillar cell. Scale bars: $20 \mu \mathrm{m}$ (A,D,F,G), $10 \mu \mathrm{m}(\mathrm{B}, \mathrm{H}), 2 \mu \mathrm{m}(\mathrm{C}, \mathrm{I}), 5 \mu \mathrm{m}(\mathrm{E})$.

most region of the outer sulcus had extended further out from the basilar membrane (Fig. 7D,G). It reached to the level of the Reissner's membrane and extended all the way across from the outer sulcus to the spiral limbus and inner sulcus. It surrounded the disorganized tectorial membrane which was often displaced towards the lateral side of the scala media, that is to the side opposite to that of its normal location (Fig. 7G). However, on the basilar membrane, recognizable inner and outer pillar cells with apparently normal morphology and in their arched configuration were maintained in position (Fig. 7H). Deiters' cells, identifiable by the presence of distinct microtubule bundles (Fig. 7I), were also in place attached to the basilar membrane (Fig. 7H,I), but they had lost their phalangeal processes and were rounded up.

\section{Peculiarities of lesion closure are specific to $\mathrm{Cx30}$ null animals}

To determine whether the particular features of lesion closure observed were specific to $\mathrm{Cx} 30$ null animals we examined the cochleae of a number of different mouse mutants in which there is a similar progressive, 'uninduced' hair cell death. The stereotypical pattern was observed in all of them, for example as shown above, mice that are null for the protein tyrosine phosphatase receptor type q $\left(\right.$ Ptprq $\left.^{-/-}\right)$(Goodyear et al., 2003) (Fig. 5C). The generation of a flat squamous-like epithelium by migration of cells from the outer side of the organ of Corti across the Deiters' cells (Taylor et al., 2012) was also observed with all other mutants examined including as previously noted those with non-functional Cx26, both the conditional knockout animals (Sun et al., 2009) and the CX26R75W transgenic animal (Fig. 6C).

\section{Discussion}

The loss of Cx30 from the Deiters' cells reduces gap junction size and the level of intercellular communication. This coincides with disruption of the normal pattern of repair at the apical surface of the organ of Corti. Normally, several of the supporting cells in immediate contact with a dying $\mathrm{OHC}$ jointly close the lesion, but 
in the Cx30 null animals, while an apparently effective repair of the epithelial surface is accomplished, only a single supporting cell participates. Thus, it seems likely that gap junctions play a role in mediating the signalling necessary to coordinate the responses of the supporting cells during lesion closure, but that intercellular communication via gap junctions is not required to trigger the supporting cells' initial response to OHC injury.

Unusually, the inner pillar cell also participated in lesion closure. Dye transfer studies have shown that while the outer pillar cells and the Deiters' cells are extensively coupled together, there is no intercellular communication between these cells and the inner pillar cells (Jagger and Forge, 2006). The first row $\mathrm{OHC}$ contacts the inner pillar cell only at the tight-adherens junction. Thus, the signal from the dying $\mathrm{OHC}$ that elicits the response from the inner pillar cell may be at that junction. Unusually extensive adherens junctions (Nunes et al., 2006) are the only contacts between an $\mathrm{OHC}$ and its immediate Deiters' or outer pillar cell neighbours throughout the normal organ of Corti and thus is a likely signalling site to trigger supporting cell responses. If this is so, it may be that the signal is not delivered equally to all cells since only one of the contacting cells, apparently at random, may respond when the normal patterns of intercellular communication are disrupted by loss of $\mathrm{Cx} 30$. That the inner pillar cell was able to effect lesion repair when normally it does not do so also indicates that this cell type retains a latent, usually redundant, capacity to expand in response to signals associated with a dying $\mathrm{OHC}$ in a manner similar to its other supporting cell cousins. It may be that the normal pathways of intercellular communication provide signals that interact in some way with adherens junction signalling to inhibit potential repair responses by inner pillar cells when $\mathrm{OHC}$ are lost.

Although the heads of the supporting cells in the Cx30 null mice retain an ability to expand to close lesions, albeit in an uncoordinated fashion, surprisingly there was no concomitant widening of the phalangeal processes of the Deiters' cells as normally occurs. The mechanisms underlying the rapid shape change of Deiters' cells following OHC loss are not known, but the present findings suggest that the expansion of the head region of the supporting cell - that is that part of the cell enclosed by the tight/adherens junction - is a separate event from the widening of the phalangeal process, and that loss of Cx30 is associated with failure of cell shape change amongst Deiters' cells in the repairing epithelium after hair cell loss. We have shown previously (Taylor et al., 2012) that extensive intercellular communication between Deiters' cells is maintained during the cell shape changes that normally occur during organ of Corti repair. The present observations therefore suggest that these shape changes may be dependent upon intercellular communication amongst the Deiters' cell population and/or from signals deriving from Hensen's cells: in Cx30 null animals Hensen's and Deiters' cells are not coupled together as they are in normal animals. Furthermore, as well as the lack of change in cell shape there was also loss of the migratory activity of Deiters' cells that we have previously observed during the cellular reorganization that follows the initial epithelial repair and which culminates in the replacement of the columnar epithelium of the organ of Corti with a 'flat' squamous-like epithelium (Taylor et al., 2012). The change in cell shape may therefore be a necessary prelude for subsequent migration.

The flat, squamous-like epithelium that normally ultimately replaces the columnar supporting cells following hair cell loss derives from a coordinated inward migration of the layer of cells from the outer side of the organ of Corti (Taylor et al., 2012). In the Cx30 null animals this layer moves inwards, but rather than generating a flat epithelium covering the basilar membrane, it eventually results in the formation of a thin layer of cells detached from the basilar membrane across the whole width of the scala media at a level above that of the normal surface of the organ of Corti. The movement of this cell layer may suggest that signals initiating migration are retained in the Cx30 null animals and that the machinery generating cell motion resides within that population of simple epithelial cells to the outerside of the organ of Corti. These cells normally express Cx26 extensively and that expression is retained when Cx30 is absent. Even though Cx30 is also normally expressed by these cells, the retention of a high level of Cx26 expression and of extensive intercellular communication likely provides sufficient cell-cell coupling for co-ordinating the activities of the cells in the absence of Cx30. Some features of the re-organisation of the organ of Corti bear similarities with the progression of wound healing in skin where cellular migration plays a crucial role. It has been reported that in mice there is upregulation of $\mathrm{Cx} 30$ at the migratory leading edge of the closing wound (Coutinho et al., 2003) leading to the proposal that $\mathrm{Cx} 30$ may be important for synchronizing cell movements during wound healing.

Loss of Cx30 did not completely eliminate gap junction plaques from the Deiters' cells nor totally abolish intercellular communication between them. It did however abolish dye transfer between Deiters' and Hensen's cells almost completely. Both $\mathrm{Cx} 30$ and $\mathrm{Cx} 26$ are present in gap junctions between Deiters' cells possibly in heteromeric configuration (Ahmad et al., 2003; Forge et al., 2003; Jagger and Forge, 2006). Immunolabelling suggests that the small gap junction plaques that are present on the membranes of Deiters' cells in the Cx30 null mice are composed of $\mathrm{Cx} 26$, and there was no evidence for its upregulation, nor for the upregulation of some other connexin, with loss of Cx30. The permeability properties of gap junction channels that contain $\mathrm{Cx} 30$, either alone or in heteromeric configuration with $\mathrm{Cx} 26$, are different from those formed of Cx26 only (Manthey et al., 2001; Marziano et al., 2003; Yum et al., 2007); Cx30 appears to play a role in restricting the passage of large anionic species. Thus, in the normal functionally mature organ of Corti anionic signalling molecules, such as IP3, c-AMP and ATP, may be unable to pass easily between Deiters' cells or between Hensen's and Deiters' cells. On the other hand it has been suggested that heteromeric $\mathrm{Cx} 26 / \mathrm{Cx} 30$ channels allow passage of calcium more readily than either Cx26 only or $\mathrm{Cx} 30$ only channels (Sun et al., 2005; Yum et al., 2007). In cultured explants of the immature organ of Corti, ATP stimulates the propagation of calcium waves that are initiated amongst the cells to the outerside and which travel across the Deiters' cell region (Majumder et al., 2010). In agreement with our present observations of the reduced transfer of $\mathrm{Nbn}$, which is a large cation, absence of $\mathrm{Cx} 30$ in these cultures results in reduced calcium wave propagation (Ortolano et al., 2008). It has been suggested that in these explant cultures ATP released from dying hair cells binds to $\mathrm{P} 2 \mathrm{Y}_{2}$ receptors that are localised to the simple epithelial cells at the outerside of the organ of Corti, triggering calcium release and initiating the waves (Gale et al., 2004). P2X and P2Y receptors are also expressed by the Hensen's and outer sulcus cells in the mature organ of Corti (Housley et al., 2002). It could be therefore that in the mature cochlea of the $\mathrm{Cx} 30$ null 
mice, signals are initiated amongst the cells to the outer side of the organ of Corti upon death of OHC and ATP release but in the absence of intercellular communication between Hensen's and Deiters they are prevented from reaching the Deiters' cell. Consequently, these cells fail to respond thereby resulting in abnormalities of epithelial repair.

In summary, the present work, points to a particular role for Cx30 in the organ of Corti in mediating repair responses following hair cell loss. With $\mathrm{Cx} 30$ also implicated in wound healing in skin, we suggest that one role for this particular connexin is in mediating the signalling involved in repair of damaged epithelia.

\section{Materials and Methods}

\section{Animals}

All animal use was conducted under regulated procedures licenced by the UK Home Office and approved by the Animal Use Committee of UCL. Embryos of Cx30 null mice $\left(\mathrm{Gjb6}^{-1-}\right)$ were obtained from the European Mouse Mutant Archive (EMMA; http://www.emmanet.org/). These animals were originally generated in a mixed 129P2/Ola-Hsd and C57BL/6 background. They were rederived in surrogate mothers and subsequently maintained on the original background. The genotyping was as described by Teubner et al. (Teubner et al. 2003). Age matched and littermate wild type and heterozygous $g j b 6^{+-}$animals acted as controls. For some comparisons, the cochleae of mice with non-functional Cx26 were examined. Mice with an insertion of the dominant negative mutation, $\mathrm{R} 75 \mathrm{~W}$, in the human version of Gjb2 gene (Kudo et al., 2003) were obtained from Prof K Ikeda (Tohoku University). These transgenic animals were maintained in our animal facility on a C57BL/6 background. In addition, some fixed cochleae excised from animals in which $\mathrm{Cx} 26$ had been conditionally deleted from the organ of Corti (Wang et al., 2009) were supplied by Dr Xi Lin (Emory University, Atlanta). The transgenic ' $\mathrm{R} 75 \mathrm{~W}$ ' mice and the mice with conditional $\mathrm{Cx} 26$ ablation, both exhibit delayed development of the organ of Corti and are profoundly deaf (Kudo et al., 2003; Wang et al., 2009).

\section{Preparation of cochleae for examination}

Auditory bullae were isolated and the cochleae exposed. Fixative was gently perfused into the cochlea through openings made by removing a small piece of bone at the apex and by rupturing the bone between the round and oval windows at the base. For immunolabelling the fixative was freshly prepared $4 \%$ paraformaldehyde in phosphate buffered saline (PBS). For scanning electron microscopy (SEM), for transmission electron microscopy (TEM) of thin-sections and for freeze fracture the fixative was $2.5 \%$ glutaraldehyde in $0.1 \mathrm{M}$ cacodylate buffer pH 7.3 with $2 \mathrm{mM} \mathrm{CaCl}_{2}$. Fixation was continued for 2 hours. The cochleae were then decalcified in 4\% EDTA for 48 hours.

\section{Immunolabelling}

Immunolabelling was performed on frozen sections of entire decalcified cochleae or whole mount preparations of the organ of Corti isolated from decalcified cochleae. Frozen sections were cut at 10-15 $\mu \mathrm{m}$. Samples for labelling were permeabilized in $0.5 \%$ Triton X-100 (Sigma), then incubated in a blocking solution of $10 \%$ goat serum in PBS for $30-60$ minutes. Samples were incubated in primary antibody in $100 \mathrm{mM}$ lysine in PBS either for 2 hours at room temperature or overnight at $4^{\circ} \mathrm{C}$. Following extensive washing in PBS, samples were incubated in the appropriate anti-mouse or anti-rabbit secondary antibody tagged to FITC or TRITC. Fluorescently conjugated phalloidin at $1 \mu \mathrm{g} / \mathrm{ml}$ was added to the secondary antibody mixture. Primary antibodies used were a mouse monoclonal to $\mathrm{Cx} 30$ (Invitrogen 33-250), a rabbit polyclonal to Cx30 (Invitrogen 71-2200), a mouse monoclonal to Cx26 (Invitrogen 33-5800), a rabbit polyclonal to Cx26 (Gap28H, a kind gift from Prof Howard Evans, University of Cardiff) and a rabbit polyclonal to Cx43 (Sigma, C6219). All of these antibodies were tested for specificity in HeLa cells expressing either Cx30, Cx26 or Cx43. No crossreactivity of the $\mathrm{Cx} 26$ or $\mathrm{Cx} 30$ antibodies was detected, and neither of the antibodies to $\mathrm{Cx} 30$ labelled tissue from the $\mathrm{Cx} 30$ null mice.

\section{Electron microscopy}

Glutaraldehyde-fixed, decalcified cochleae for SEM and for sectioning were postfixed in $1 \%$ cacodylate-buffered $\mathrm{OsO}_{4}$. Cochleae for SEM were dissected to isolate the organ of Corti in approximately half turn segments which were processed through the thiocarbohydrazide- $\mathrm{OsO}_{4}$ repeated procedure (Davies and Forge, 1987 ) before dehydration in an ethanol series and critical point drying. They were examined in a JEOL 6700F cold field emission instrument operating (JEOL UK, Welwyn Garden City) at 3 or $5 \mathrm{Kv}$ and digital images were collected. Cochleae for sectioning were processed intact without any dissection. Following post-fixation in $\mathrm{OsO}_{4}$ they were partially dehydrated to $70 \%$ ethanol then incubated in a saturated solution of uranyl acetate in $70 \%$ ethanol overnight at $4{ }^{\circ} \mathrm{C}$ before completion of dehydration and embedding in plastic. Sections of the entire cochlea for light microscopy, ca. $1 \mu \mathrm{m}$ thick, were cut parallel to the modiolus and stained with Toluidine Blue. A series of thin sections for TEM were then cut. For each cochlea that was prepared for thin-sectioning, the opposite ear of the same animal was prepared for SEM. For freeze-fracture, segments of the organ of Corti were dissected from glutaraldehyde-fixed decalcified cochleae, and cryoprotected in $25 \%$ glycerol in cacodylate buffer before freezing in rapidly stirred propaneisopentane $(4: 1)$ cooled in liquid nitrogen. Freeze-fracture and replica production were performed in a Balzers BAF400D unit using procedures described elsewhere (Forge et al., 2003). Thin sections and freeze-fracture replicas were examined in a JEOL 1200EXII instrument and digital images collected with a Gatan Ultrascan camera (Gatan UK, Abingdon, UK). Images of freeze-fracture replicas are presented in reverse contrast so that shadows appear black. All digital images were adjusted for optimal contrast and brightness using Photoshop CS4 software (Adobe, San Jose, CA, USA).

\section{Dye transfer in cochlear slice preparations}

Slices of the viable cochlea (Jagger and Forge, 2006; Taylor et al., 2012) were obtained from Cx30 null animals at P15-P16. Slices were maintained in and superfused with artificial perilymph $(150 \mathrm{mM} \mathrm{NaCl}, 4 \mathrm{mM} \mathrm{KCl}, 2 \mathrm{mM} \mathrm{MgCl}$ $1.3 \mathrm{mM} \mathrm{CaCl}_{2}, 10 \mathrm{mM}$ HEPES, and $5 \mathrm{mM}$ glucose, $\mathrm{pH}$ adjusted to 7.3 with $\mathrm{NaOH}$ ). Individual supporting cells were patch clamped and neurobiotin (Nbn) was injected into them via the patch clamp electrode during whole-cell patch clamp recording. The slice was incubated at room temperature for 10 minutes and then fixed in $4 \%$ paraformaldehyde. To detect $\mathrm{Nbn}$, slices were permeabilized $(0.1 \%$ Triton $\mathrm{X}-100$ for 40 minutes $)$, blocked $\left(0.1 \mathrm{M}\right.$ L-lysine, at $35^{\circ} \mathrm{C}$ for 40 minutes), and incubated for 2 hours in Alexa-Fluor-555-conjugated streptavidin (1:1000; Invitrogen, Carlsbad, CA). The slices were also immunolabelled for Cx26 using the Gap28H antibody, and a secondary antibody conjugated to Alexa Fluor 633

\section{Acknowledgements}

We thank Prof Katsuhisa Ikeda, Juntendo University School of Medicine, Tokyo, Japan for supply of transgenic Cx26 R75W mice, Dr Xi Lin, Emory University, Atlanta USA for cochleae from conditional Cx26 mutants and Prof Guy Richardson, University of Sussex UK, for cochleae from Ptprq mutants.

\section{Author contributions}

A.F, D.J.J., J.J.K. and R.R.T. contributed equally to the design of the research, performing experiments and analysing data.

\section{Funding}

The work was supported by a project grant from the Biotechnology and Biological Sciences Research Council (BBSRC) [project grant number BB/D009669/1 to A.F. and D.J.J.]; the Rosetrees Trust through Deafness Research UK [grant number 294:ILO:AF]; a studentship for J.J.K. from Deafness Research UK [grant number 403.EIP.DM]; and a project grant from Deafness Research UK [grant number 561:UEI:DJ to D.J.J.] .

\section{References}

Abrashkin, K. A., Izumikawa, M., Miyazawa, T., Wang, C. H., Crumling, M. A. Swiderski, D. L., Beyer, L. A., Gong, T. W. and Raphael, Y. (2006). The fate of outer hair cells after acoustic or ototoxic insults. Hear. Res. 218, 20-29.

Ahmad, S., Chen, S., Sun, J. and Lin, X. (2003). Connexins 26 and 30 are coassembled to form gap junctions in the cochlea of mice. Biochem. Biophys. Res. Commun. 307, 362-368.

Bedner, P., Steinhäuser, C. and Theis, M. (2012). Functional redundancy and compensation among members of gap junction protein families? Biochim. Biophys. Acta 1818, 1971-1984.

Cohen-Salmon, M., Maxeiner, S., Krüger, O., Theis, M., Willecke, K. and Petit, C. (2004). Expression of the connexin43- and connexin45-encoding genes in the developing and mature mouse inner ear. Cell Tissue Res. 316, 15-22.

Coutinho, P., Qiu, C., Frank, S., Tamber, K. and Becker, D. (2003). Dynamic changes in connexin expression correlate with key events in the wound healing process. Cell Biol. Int. 27, 525-541.

Davies, S. and Forge, A. (1987). Preparation of the mammalian organ of Corti for scanning electron microscopy. J. Microsc. 147, 89-101.

Forge, A. (1985). Outer hair cell loss and supporting cell expansion following chronic gentamicin treatment. Hear. Res. 19, 171-182.

Forge, A., Becker, D., Casalotti, S., Edwards, J., Marziano, N. and Nevill, G. (2003). Gap junctions in the inner ear: comparison of distribution patterns in differen 
vertebrates and assessement of connexin composition in mammals. J. Comp. Neurol. 467, 207-231.

Gale, J. E., Piazza, V., Ciubotaru, C. D. and Mammano, F. (2004). A mechanism for sensing noise damage in the inner ear. Curr. Biol. 14, 526-529.

Goodyear, R. J., Legan, P. K., Wright, M. B., Marcotti, W., Oganesian, A., Coats, S. A., Booth, C. J., Kros, C. J., Seifert, R. A., Bowen-Pope, D. F. et al, (2003). A receptor-like inositol lipid phosphatase is required for the maturation of developing cochlear hair bundles. J. Neurosci. 23, 9208-9219.

Hawkins, J. E. and Engstrom, H. (1963). Effect of kanamycin on cochlear cytoarchitecture. Acta Otolaryngol. 188 Suppl., 100-107.

Hirt, B., Gleiser, C., Eckhard, A., Mack, A. F., Müller, M., Wolburg, H. and Löwenheim, H. (2011). All functional aquaporin-4 isoforms are expressed in the rat cochlea and contribute to the formation of orthogonal arrays of particles. Neuroscience 189, 79-92.

Housley, G. D., Jagger, D. J., Greenwood, D., Raybould, N. P., Salih, S. G., Järlebark, L. E., Vlajkovic, S. M., Kanjhan, R., Nikolic, P., Muñoz, D. J. et al. (2002). Purinergic regulation of sound transduction and auditory neurotransmission. Audiol. Neurootol. 7, 55-61.

Jagger, D. J. and Forge, A. (2006). Compartmentalized and signal-selective gap junctional coupling in the hearing cochlea. J. Neurosci. 26, 1260-1268.

Kanaporis, G., Brink, P. R. and Valiunas, V. (2011). Gap junction permeability: selectivity for anionic and cationic probes. Am. J. Physiol. Cell Physiol. 300, C600C609.

Kretz, M., Euwens, C., Hombach, S., Eckardt, D., Teubner, B., Traub, O., Willecke, K. and Ott, T. (2003). Altered connexin expression and wound healing in the epidermis of connexin-deficient mice. J. Cell Sci. 116, 3443-3452.

Kudo, T., Kure, S., Ikeda, K., Xia, A. P., Katori, Y., Suzuki, M., Kojima, K., Ichinohe, A., Suzuki, Y., Aoki, Y. et al. (2003). Transgenic expression of a dominant-negative connexin26 causes degeneration of the organ of Corti and nonsyndromic deafness. Hum. Mol. Genet. 12, 995-1004.

Majumder, P., Crispino, G., Rodriguez, L., Ciubotaru, C. D., Anselmi, F., Piazza, V., Bortolozzi, M. and Mammano, F. (2010). ATP-mediated cell-cell signaling in the organ of Corti: the role of connexin channels. Purinergic Signal. 6, 167-187.

Manthey, D., Banach, K., Desplantez, T., Lee, C. G., Kozak, C. A., Traub, O., Weingart, R. and Willecke, K. (2001). Intracellular domains of mouse connexin26 and -30 affect diffusional and electrical properties of gap junction channels. J. Membr. Biol. 181, 137-148.

Marziano, N. K., Casalotti, S. O., Portelli, A. E., Becker, D. L. and Forge, A. (2003). Mutations in the gene for connexin 26 (GJB2) that cause hearing loss have a dominant negative effect on connexin 30. Hum. Mol. Genet. 12, 805-812.
McDowell, B., Davies, S. and Forge, A. (1989). The effect of gentamicin-induced hair cell loss on the tight junctions of the reticular lamina. Hear. Res. 40, 221-232.

Nunes, F. D., Lopez, L. N., Lin, H. W., Davies, C., Azevedo, R. B., Gow, A. and Kachar, B. (2006). Distinct subdomain organization and molecular composition of a tight junction with adherens junction features. J. Cell Sci. 119, 4819-4827.

Ortolano, S., Di Pasquale, G., Crispino, G., Anselmi, F., Mammano, F. and Chiorini, J. A. (2008). Coordinated control of connexin 26 and connexin 30 at the regulatory and functional level in the inner ear. Proc. Natl. Acad. Sci. USA 105, 18776-18781.

Qu, Y., Tang, W., Zhou, B., Ahmad, S., Chang, Q., Li, X. and Lin, X. (2012). Early developmental expression of connexin26 in the cochlea contributes to its dominate functional role in the cochlear gap junctions. Biochem. Biophys. Res. Commun. 417, 245-250.

Raphael, Y. and Altschuler, R. A. (1991). Scar formation after drug-induced cochlear insult. Hear. Res. 51, 173-183.

Sun, J., Ahmad, S., Chen, S., Tang, W., Zhang, Y., Chen, P. and Lin, X. (2005). Cochlear gap junctions coassembled from $\mathrm{Cx} 26$ and 30 show faster intercellular $\mathrm{Ca} 2+$ signaling than homomeric counterparts. Am. J. Physiol. Cell Physiol. 288, C613C623.

Sun, Y., Tang, W., Chang, Q., Wang, Y., Kong, W. and Lin, X. (2009). Connexin30 null and conditional connexin26 null mice display distinct pattern and time course of cellular degeneration in the cochlea. J. Comp. Neurol. 516, 569-579.

Taylor, R. R., Nevill, G. and Forge, A. (2008). Rapid hair cell loss: a mouse model for cochlear lesions. J. Assoc. Res. Otolaryngol. 9, 44-64.

Taylor, R. R., Jagger, D. J. and Forge, A. (2012). Defining the cellular environment in the organ of Corti following extensive hair cell loss: a basis for future sensory cell replacement in the Cochlea. PLoS ONE 7, e30577.

Teubner, B., Michel, V., Pesch, J., Lautermann, J., Cohen-Salmon, M., Söhl, G., Jahnke, K., Winterhager, E., Herberhold, C., Hardelin, J. P. et al. (2003). Connexin30 (Gjb6)-deficiency causes severe hearing impairment and lack of endocochlear potential. Hum. Mol. Genet. 12, 13-21.

Wang, Y., Chang, Q., Tang, W., Sun, Y., Zhou, B., Li, H. and Lin, X. (2009). Targeted connexin26 ablation arrests postnatal development of the organ of Corti. Biochem. Biophys. Res. Commun. 385, 33-37.

Wangemann, P. (2002). K+ cycling and the endocochlear potential. Hear. Res. 165, 1-9.

Yum, S. W., Zhang, J., Valiunas, V., Kanaporis, G., Brink, P. R., White, T. W. and Scherer, S. S. (2007). Human connexin26 and connexin 30 form functional heteromeric and heterotypic channels. Am. J. Physiol. Cell Physiol. 293, C1032C1048. 